\title{
Real scalar field kinks and antikinks and their perturbation spectra in a closed universe
}

\author{
Betti Hartmann, ${ }^{1,2}$ Gabriel Luchini, ${ }^{3}$ Clisthenis P. Constantinidis $\odot,{ }^{3}$ and Carlos F. S. Pereira ${ }^{3}$ \\ ${ }^{1}$ Instituto de Física de São Carlos (IFSC), Universidade de São Paulo (USP), \\ CP 369, 13560-970 São Carlos, São Paulo, Brazil \\ ${ }^{2}$ Department of Theoretical Physics, University of the Basque Country UPV/EHU, 48080 Bilbao, Spain \\ ${ }^{3}$ Departamento de Física, Universidade Federal do Espírito Santo (UFES), \\ CEP 29075-900 Vitória, Espírito Santo, Brazil
}

(Received 20 November 2019; accepted 17 March 2020; published 3 April 2020)

\begin{abstract}
We demonstrate that solitons of a simple real scalar field model that are static and linearly stable do exist when considered in a $(3+1)$-dimensional, spatially compact space-time background, the static Einstein universe, which is a good approximation to the observed Universe for sufficiently small time intervals. We study the properties of these solutions for a $\Phi^{4}$-potential and demonstrate that next to the fundamental solutions, excited configurations exist. We also investigate general perturbations about the solitons, determine their eigenfrequency spectra, and compare them to those of the perturbations about the vacua of the model. We find that the degeneracy with respect to the multipoles of the perturbation, which is present for the vacua, no longer exists in the presence of the soliton. Moreover, specific perturbations correspond to zero modes of the system. Our results have applications in condensed matter physics as well as computations of quantum effects (e.g., the Casimir energy) in spatially compact space-times in the presence of solitonlike objects.
\end{abstract}

DOI: 10.1103/PhysRevD.101.076004

\section{INTRODUCTION}

After the discovery $[1,2]$ of the Englert-Brout-HiggsGuralnik-Hagen-Kibble boson [3-5] in 2012, a fundamental scalar field in nature, the study of scalar fields in diverse contexts of physics has become of interest again. Scalar fields had previously been present in a number of high and low energy physics models, such as in the theory of inflation in the primordial universe that is supposed to be driven by a slow-roll scalar field, as well as in condensed matter, where collective phenomena are often effectively described by a scalar field. The typical example for the latter case is the Ginzburg-Landau model [6] describing superconductivity.

So-called solitonic solutions of these field theory models are of particular interest, especially due to their possible interpretation as relativistic particles [7]. The existence of such configurations is limited by some important features of the model, e.g., its integrability [8] and/or the existence of a topological degree characterizing the field as a topological soliton [9]. Besides, important restrictions

Published by the American Physical Society under the terms of the Creative Commons Attribution 4.0 International license. Further distribution of this work must maintain attribution to the author(s) and the published article's title, journal citation, and DOI. Funded by SCOAP . are imposed by the dimensionality of space and the types of fields which are present in the model with the possibility of finding a static solitonic solution; this is given by Derrick's theorem [10]. For theories in $(d+1)$-dimensional Minkowski space-time with only scalar fields and no terms involving derivatives higher than second order, this theorem predicts that only in $d=1$ or $d=2$ can static solitons be found. However, even in flat space-time, there are ways to evade this theorem, as in the case of $Q$-balls [11-13], which are nontopological solitonic scalar field configurations that exist in more than one spatial dimension and possess a time-dependent phase. In $(3+1)$-dimensional Minkowski space-time, the scalar field model needs (at least) a sixth order self-interaction potential for $Q$-balls to exist [13-16]. When extending these models to curved space-time, self-gravitating counterparts of $Q$-balls, socalled boson stars exist [15-21]. In curved space-time, no general direct extension of Derrick's theorem seems to restrict the solitonic solutions, and the possibility of finding them is open for exploration, although there has been recent progress for asymptotically flat space-times [22].

In this paper, we study a real scalar field model in a $(3+1)$-dimensional Einstein universe which is spatially compact. This model was previously studied in [23], but only for one very specific case. The Einstein universe describes a universe filled with a perfect fluid that has positive energy density and vanishing pressure. In addition, 
there is a positive cosmological constant that allows for the existence of a static solution to the Einstein equation. While it is well known that the Universe is acceleratingly expanding and, as such, contains a substantial amount of dark energy that can mathematically be described by a positive cosmological constant, the Einstein universe is often used as an approximation to the "real Universe" for sufficiently small time intervals, which makes, e.g., computations of quantum states feasible (see, e.g., [24-27]). Moreover, the compactness of this space-time is of interest in condensed matter physics, in particular, in Bose-Einstein condensation (see, e.g., [28-31]) since it is well known that the geometry of a finite system plays an important role in the thermal behavior of the bosons and the actual condensation process.

Here, we extend the analysis of [23] considerably to show that additional solutions with interesting properties exist. Moreover, we study general linear perturbations about these solutions and compute the eigenfrequency spectra. The outline of our manuscript is as follows. We give the model in Sec. II, while we discuss the numerical solutions of the model in Sec. III. In Sec. IV, we present our results on the eigenfrequency spectra of the perturbations about the solutions obtained in Sec. III. We conclude in Sec. V.

\section{THE MODEL}

We study a self-interacting scalar field model in $(3+1)$ dimensions with action given by

$$
\begin{aligned}
S & =\int \mathrm{d}^{4} x \sqrt{-g} \mathcal{L} \\
& =\int \mathrm{d}^{4} x \sqrt{-g}\left(-\frac{1}{2} \partial_{\mu} \Phi^{*} \partial^{\mu} \Phi-V(|\Phi|)\right), \\
V(|\Phi|) & =\frac{\lambda}{4}\left(|\Phi|^{2}-\eta^{2}\right)^{2},
\end{aligned}
$$

where $g$ denotes the determinant of the metric tensor $g_{\mu \nu}$ of the $(3+1)$-dimensional space-time background, which we assume to be nondynamical. The self-coupling constant $\lambda$ is chosen positive such that the potential is positive definite, i.e., has its lowest value $V \equiv 0$ at what we will refer to as the "vacua" in the following, i.e., at $|\phi|_{0}= \pm \eta$. A priori, we allow the scalar field to be complex valued, and hence the star denotes complex conjugation.

The equation of motion following from the variation of the action (1) with respect to the scalar field reads

$$
\begin{aligned}
\frac{1}{\sqrt{-g}} \partial_{\mu}\left(\sqrt{-g} \partial^{\mu} \Phi\right)-2 \frac{\partial V}{\partial|\Phi|^{2}} \Phi & =0 \\
\Rightarrow \quad \frac{1}{\sqrt{-g}} \partial_{\mu}\left(\sqrt{-g} \partial^{\mu} \Phi\right)-\lambda\left(|\Phi|^{2}-\eta^{2}\right) \Phi & =0,
\end{aligned}
$$

while we assume the space-time background to be fixed. In the following, we will choose the static Einstein universe [32] with metric

$$
d s^{2}=-d t^{2}+R_{0}^{2}\left[d \chi^{2}+\sin ^{2} \chi\left(d \theta^{2}+\sin ^{2} \theta d \varphi^{2}\right)\right],
$$

where the coordinates $(t, \chi, \theta, \varphi)$ have the following ranges: $t \in]-\infty: \infty[, \chi \in[0: \pi], \theta \in[0: \pi], \varphi \in[0: 2 \pi[$. Constant $t$-sections are three-spheres with radius $R_{0}$; i.e., the topology of this compact space-time is $\mathbb{R} \times S^{3}$. The metric given by Eq. (3) is a Friedman-Lemâitre-Robertson-Walker (FLRW) space-time and a solution to the Einstein equation $G_{\mu \nu}+\Lambda g_{\mu \mu}=8 \pi \operatorname{diag}(\rho,-p,-p,-p)$, where $\rho=\Lambda / 4$, $p=0$ are the energy-momentum components of a perfect fluid with energy density $\rho$ and pressure $p$. Moreover, the cosmological constant $\Lambda=R_{0}^{-2}$ is positive. While the observed Universe is known to be accelerately expanding, the Einstein universe is normally considered a good approximation to the FLRW space-time in sufficiently small time intervals and has frequently been considered in computations of the quantum vacuum energy in the Universe (see, e.g., [25]).

In the following, we will demonstrate that next to the vacuum solution $|\Phi|_{0} \equiv \pm \eta$, this model contains nontrivial, localized, and static solutions that resemble kinks and antikinks. In the following, we will hence assume that $\Phi(\vec{r}, t) \sim \phi(\chi)$, i.e., that the scalar field depends only on $\chi$. Note that in Minkowski space-time the existence of real, static scalar field solutions of this simple model is forbidden by Derrick's theorem [10]. Moreover, in a curved space-time background, solutions do not exist if the spacetime is asymptotically flat [22]. However, in the spatially compact space-time that we are studying here, the additional length scale - the radius of the three-sphere $R_{0}$ leads to the existence of solitonic solutions. This was shown in [23] for the $\Phi^{4}$-potential, albeit only for one specific value of the radius (in relation to the mass of the scalar field). Note that scalar field solitons on an $S^{3}$, i.e., in a compact space, were also studied previously in a nonlinear sigma model [33].

With these assumptions, the nonvanishing components of the energy-momentum tensor $T_{\mu \nu}=g_{\mu \nu} \mathcal{L}-2 \frac{\partial \mathcal{L}}{\partial g^{\mu \nu}}$ read

$T_{t}^{t}=T_{\theta}^{\theta}=T_{\varphi}^{\varphi}=-\frac{1}{2} \frac{\phi^{2}}{R_{0}^{2}}-V(\phi), \quad T_{\chi}^{\chi}=\frac{1}{2} \frac{\phi^{2}}{R_{0}^{2}}-V(\phi)$,

where the prime now and in the following denotes the derivative with respect to $\chi$. The energy density of the solutions is given by $\varepsilon=-T_{t}^{t}$, and associating the Noether charge related to the time-translation invariance to the total conserved energy $E$ of the solution, we can write

$$
\begin{aligned}
E & =\int \mathrm{d}^{3} x \sqrt{-g} \varepsilon \\
& =4 \pi R_{0}^{3} \int_{0}^{\pi} \mathrm{d} \chi \sin ^{2} \chi\left(\frac{1}{2} \frac{\phi^{\prime 2}}{R_{0}^{2}}+\frac{\lambda}{4}\left(\phi^{2}-\eta^{2}\right)^{2}\right) .
\end{aligned}
$$

As is obvious from the expression, we do not need to require the solutions to tend to the vacua of the potential to 
obtain finite energy solutions, which would be necessary in Minkowski or asymptotically Minkowski space-time. In fact, we will show below that the values of $\phi(\chi=0)$ and $\phi(\chi=\pi)$, respectively, depend solely on the ratio between $R_{0}$ and the relevant length/mass scale in the model.

\section{NONTRIVIAL SOLUTIONS}

Rescaling $\phi(\chi) \rightarrow \eta \phi(\chi)$, the equation of motion for the scalar field reads

$$
\phi^{\prime \prime}+2 \cot \chi \phi^{\prime}-\alpha \phi\left(\phi^{2}-1\right)=0,
$$

where $\alpha=\lambda \eta^{2} R_{0}^{2}$ is the only dimensionless parameter and corresponds to the ratio between the radius of the threesphere and the core radius of the solution $\sim(\sqrt{\lambda} \eta)^{-1}$. Equation (6) cannot be solved analytically requiring nontriviality. We have hence employed a numerical technique [34] and used the following boundary conditions to insure regularity of the solutions at $\chi=0$ and $\chi=\pi$ :

$$
\phi^{\prime}(\chi=0)=\phi^{\prime}(\chi=\pi)=0 .
$$

Equation (6) was solved numerically in [23] - however, only for a very specific value of $\alpha \equiv 4$. In the following, we will discuss the behavior of the solutions for generic values of $\alpha$ and refer to solutions tending from a positive value at $\chi=0$ to a negative value at $\chi=\pi$ as antikinks, while the corresponding kinks are obtained easily by letting $\phi \rightarrow-\phi$, which is clearly a symmetry of Eq. (6).

The dimensionless energy density $\tilde{\varepsilon}$ and dimensionless energy $\tilde{E}$ of the solutions then reads

$\tilde{\varepsilon}=\frac{1}{2} \phi^{\prime 2}+\frac{\alpha}{4}\left(\phi^{2}-1\right)^{2}, \quad \tilde{E}=4 \pi \int_{0}^{\pi} \mathrm{d} \chi \sin ^{2} \chi \tilde{\varepsilon}$,

with $\varepsilon=\eta^{2} \tilde{\varepsilon} / R_{0}^{2}$ and $E=R_{0} \eta^{2} \tilde{E}$, respectively.

Note that Eq. (6) can be rewritten in the following form:

$$
\frac{\phi^{\prime 2}}{2}-\frac{\alpha}{4}\left(\phi^{2}-1\right)^{2}=C-2 \int_{0}^{\chi} \cot \tilde{\chi} \phi^{\prime 2} \mathrm{~d} \tilde{\chi},
$$

where $C$ is an integration constant. One can construct a mechanical analog of this equation by considering $\chi$ to be "time" and $\phi$ the position describing a particle moving along a trajectory $\phi(\chi)$. Then the left-hand side of Eq. (9) can be interpreted as the sum of kinetic and potential energy $U(\phi)=-\frac{\alpha}{4}\left(\phi^{2}-1\right)^{2}$ of this particle, with $C$ being the "energy" and the integral term on the right-hand side a friction term. The presence of this friction term is essential for the existence of the nontrivial solutions that we will present below. In Fig. 1, we show $U(\phi)$ for different values of $\alpha$. This indicates - taking the periodicity of the $\chi$-direction into account-what type of solutions are possible. In contrast to flat space-time, the value of $\phi(\chi)$

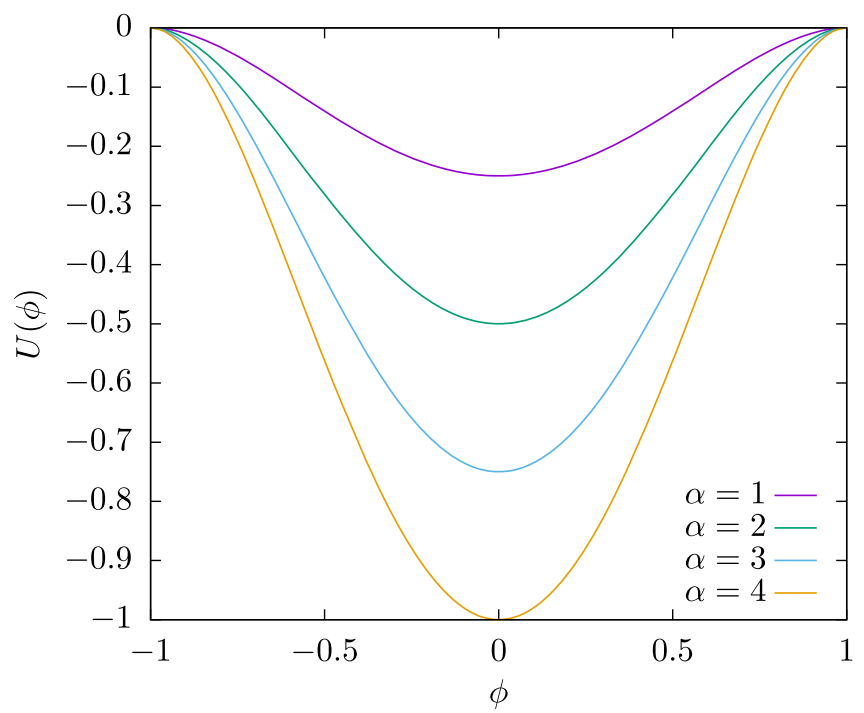

FIG. 1. We show the potential $U(\phi)=-\frac{\alpha}{4}\left(\phi^{2}-1\right)^{2}$ [see the discussion below Eq. (9)], in which the "particle" would move on a "trajectory" $\phi(\chi)$ under the influence of a friction term for different values of $\alpha$.

has to be "fine-tuned" with respect to $\alpha$ because $\alpha$ determines the slope of the potential, i.e., the value of $\phi^{\prime}$ and hence the value of the friction term in Eq. (9). Moreover, $\phi(\chi=0) \neq 1$ for $\alpha$ finite because we have the additional condition that $\phi(\chi=0)= \pm \phi(\chi=\pi)$ due to periodicity (see below for more details). In other words, for the particle to roll back to $\pm \phi(\chi=0)$ in a given $\alpha$-potential (which possesses increased slope when $\alpha$ increases), we have to choose $\phi(\chi=0)$ appropriately. Moreover, Fig. 1 indicates that given enough "initial energy," i.e., starting at $\phi(\chi=0)$ sufficiently large, the particle can role back to $\phi(\chi=\pi)=\phi(\chi=0)$ and, additionally, oscillate a number of times around $\phi=0$; i.e., we would expect configurations whose shape resembles that of a pair of antikink kinks as well as excited kinks and antikink kinks, respectively, to exist as well. Our numerical results below show that these solutions do indeed exist in this model. It is clear that the existence of these solutions is fundamentally linked to the periodicity of the $\chi$-direction as well as to the fact that we are in three spatial dimensions.

\section{A. Fundamental kinks and antikinks}

The antikinks of the $\phi^{4}$-model have been constructed in [23] for $\alpha=4$. In Fig. 2, we show the solutions to the model for different values of $\alpha$ as well as the corresponding energy densities $\tilde{\varepsilon}$. This figure demonstrates that the value of $\phi(0)$ is a function of $\alpha$. In fact, when varying the value of $\alpha$, we observe that the solutions exist only to a minimal, nonvanishing value of $\alpha=\alpha_{\min , 1}$, which we find numerically to be $\alpha_{\min , 1}=3$. The reason for this is that the value of $\phi(\chi=0)$ is a decreasing function of $\alpha$ and, for $\alpha$ sufficiently small, the kinks cease to exist. This is also obvious 

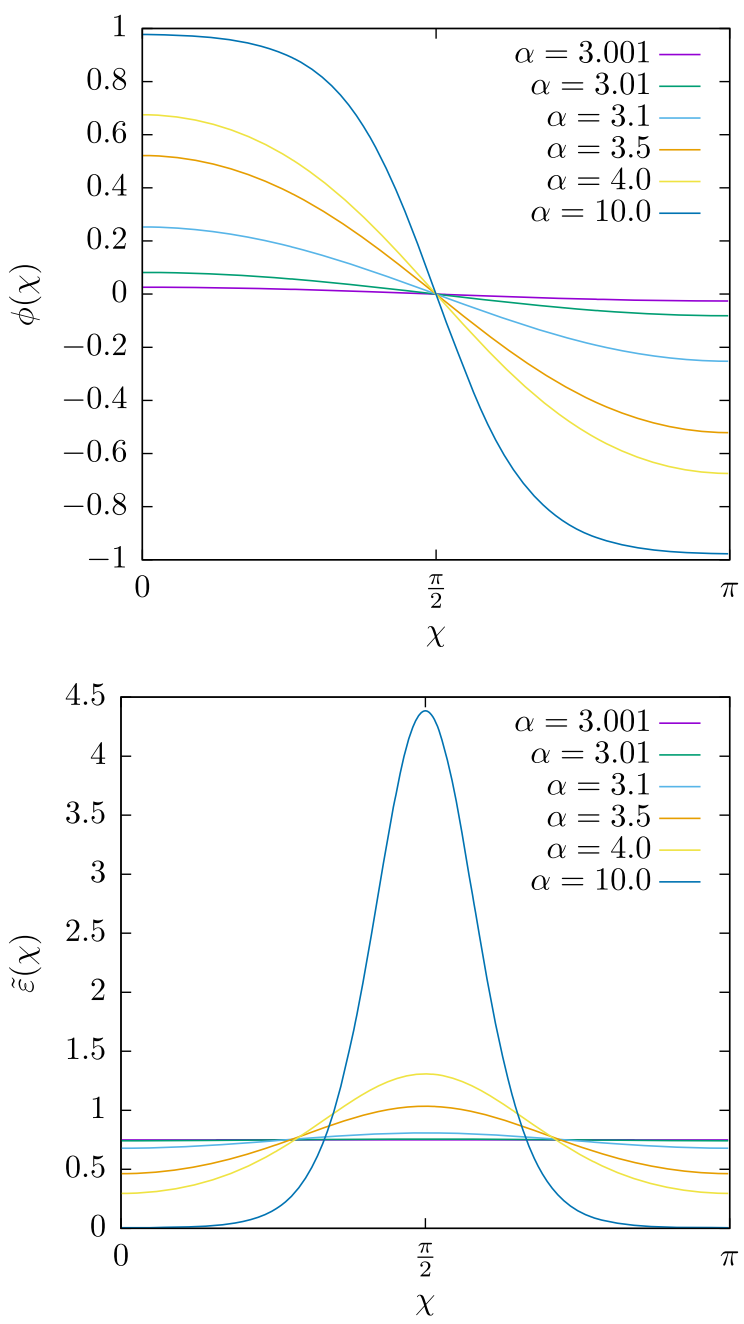

FIG. 2. We show the profile of the function $\phi(\chi)$ in (left panel) the $\phi^{4}$-model as well as (right) the energy density $\tilde{\varepsilon}$ of these solutions for different values of $\alpha$.

when considering the plots of the energy density $\tilde{\varepsilon}$. For decreasing $\alpha$, the energy density spreads over the $\chi$ direction and becomes equivalent to zero for $\alpha=\alpha_{\min , 1}$. This is related to the fact that $\alpha$ is the ratio between the width of the kink and the radius of the $S^{3}$ sphere. So, one would naturally expect that solutions exist only for a radius larger than the width of the kink. This is shown in Fig. 3, where we give the value of $\phi(\chi=0)$ in dependence of the coupling $\alpha$. Clearly for $\alpha<\alpha_{\min , 1}=3$ we find that $\phi(0)=$ 0 and hence $\phi(\chi) \equiv 0$. Increasing $\alpha$ from this value, we find that $\phi(0)$ increases up to $\phi(0)=1$, which it reaches roughly when $\alpha=\mathcal{O}(100)$.

The limit of $\alpha \rightarrow \infty$ corresponds to the limit of flat, $(3+1)$-dimensional Minkowski space-time. The existence of nontrivial, real scalar field solutions is forbidden by Derrick's theorem in this case. The approach to this limit can also be seen in Fig. 2 and is confirmed by our numerics: for $\alpha \rightarrow \infty$, the value of $\phi(0) \rightarrow 1$, but at the same time, the function $\phi(\chi)$ has an increasing derivative at $\chi=\pi / 2$. In

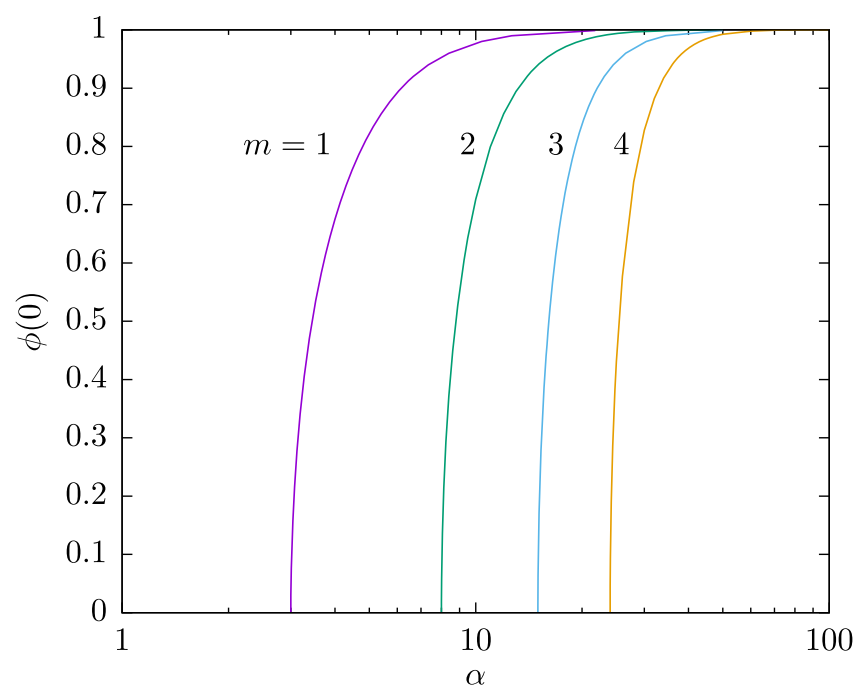

FIG. 3. We show the value of $\phi(\chi=0) \equiv \phi(0)$ as a function of $\alpha$ for the fundamental antikink solution $(m=1)$ and the kink or antikink $(m=2)$ as well as the first excited antikink $(m=3)$ and the first excited kink or antikink $(m=4)$, respectively.

the limit $\alpha \rightarrow \infty$, we expect that $\phi(\chi)=1$ for $\chi \in[0: \pi / 2[$, $\phi(\pi / 2)=0$, and $\phi(\chi)=-1$ for $\chi \in] \pi / 2: \pi]$ with an infinite derivative of $\phi$ and hence an infinite value of the energy density $\tilde{\varepsilon}$ at $\chi=\pi / 2$.

\section{B. Kinks and antikinks}

Next to the fundamental kink solution described in Eq. (3.1) for which $\phi(\chi=0)=-\phi(\chi=\pi)$, we have constructed solutions of Eq. (6) fulfilling $\phi(\chi=0)=\phi(\chi=\pi)$. The latter solutions have two nodes in the profile function $\phi(\chi)$. Using the notation of solutions of the $\phi^{4}$-model in $(1+1)$ dimensions, we refer to these solutions as kinks and antikinks. In Fig. 4, we show an example of such a solution for $\alpha=10$ together with the energy density $\tilde{\varepsilon}$.

The dependence of the value of the scalar field function $\phi(\chi=0)$ on $\alpha$ is shown in Fig. 3. Obviously, we need to choose the ratio between the width of the kink or antikink and the radius of the three-sphere larger to obtain these solutions. We find that at $\alpha_{\min , 2}=8$, the value of $\phi(\chi=0) \rightarrow$ 0 for these solutions; i.e., kinks and antikinks exist only for $\alpha \geq 8$.

\section{C. $m$-excited kinks and kinks and antikinks}

Next to the solutions described in Eqs. (3.1) and (3.2), we have constructed excited solutions of the former that differ in the number of nodes of the scalar field function $\phi(\chi)$. This is shown in Fig. 5, where we give examples of first excited antikink and the first excited kink-antikink solutions, respectively. Figure 3 further demonstrates that first excited antikinks exist only for $\alpha \geq 15$, i.e., $\alpha_{\min , 3}=15$, while first excited kinks and antikinks exist only for $\alpha \geq 24$, i.e., $\alpha_{\min , 4}=24$. The behavior of $\alpha_{\min , m}$ can be explained easily 

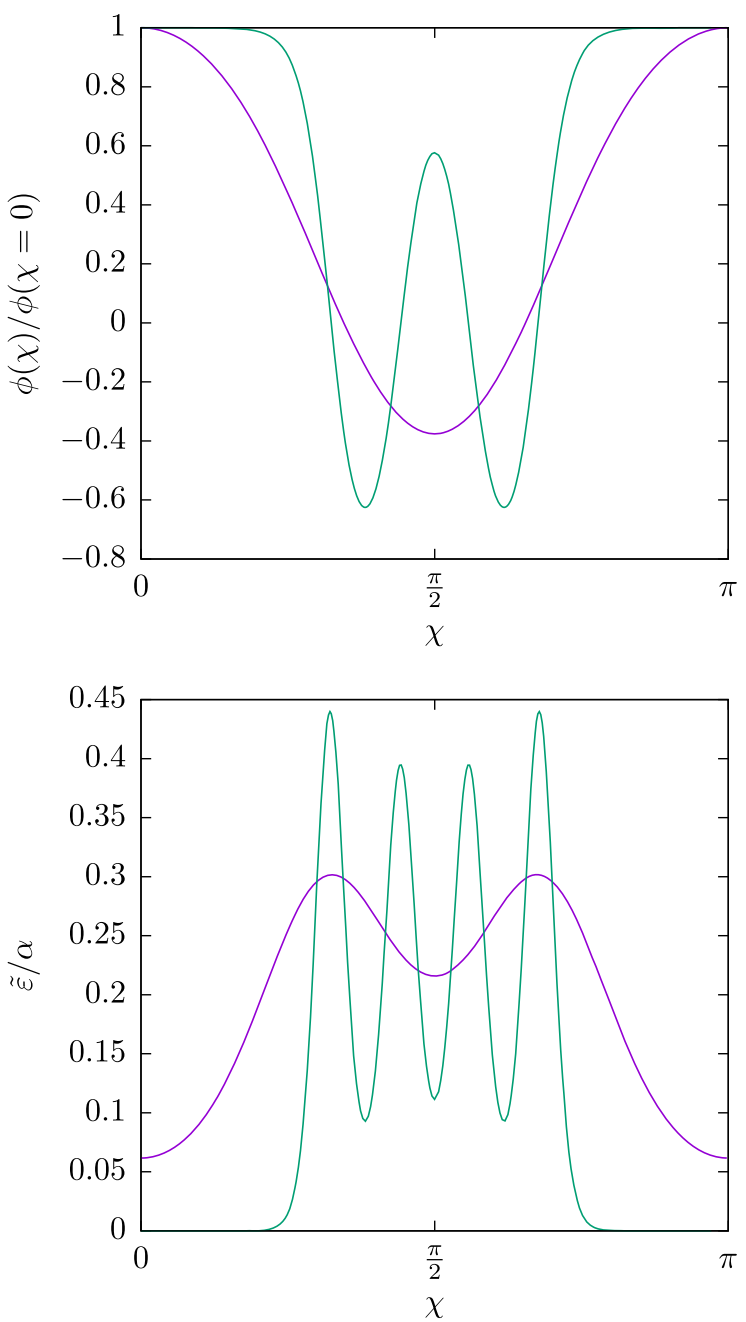

FIG. 4. (Left panel) We show the profile of a fundamental kinkantikink solution as well as its energy density $\tilde{\varepsilon}$ for $\alpha=10.0$. The profile function $\phi(\chi)$ of this solution has $m=2$ nodes (purple lines). We also show the first excited kink-antikink solution and its energy density $\tilde{\varepsilon}$ for $\alpha=100.0$ (green lines). Note that this solution possesses $m=4$ nodes in the profile function $\phi(\chi)$.

when considering the small $\phi$ limit of Eq. (6). In this limit, we can drop the $\phi^{3}$ term and the equation becomes linear. Changing coordinates to $z=\cos \chi$, the equation then becomes a hypergeometric equation that has regular solutions in the form of Gegenbauer polynomials if

$$
\alpha_{m}=m(m+2), \quad m=1,2,3,4, \ldots
$$

This relation gives exactly the values of $\alpha_{\min , m}$ that we find numerically when $\phi(\chi=0) \rightarrow 0$.

\section{PERTURBATIONS AND THEIR SPECTRA}

The aim of our study of perturbations around the constructed solutions is twofold: (a) since no-go theorems related to real scalar field solitons often require the additional property of stability, we will study whether the
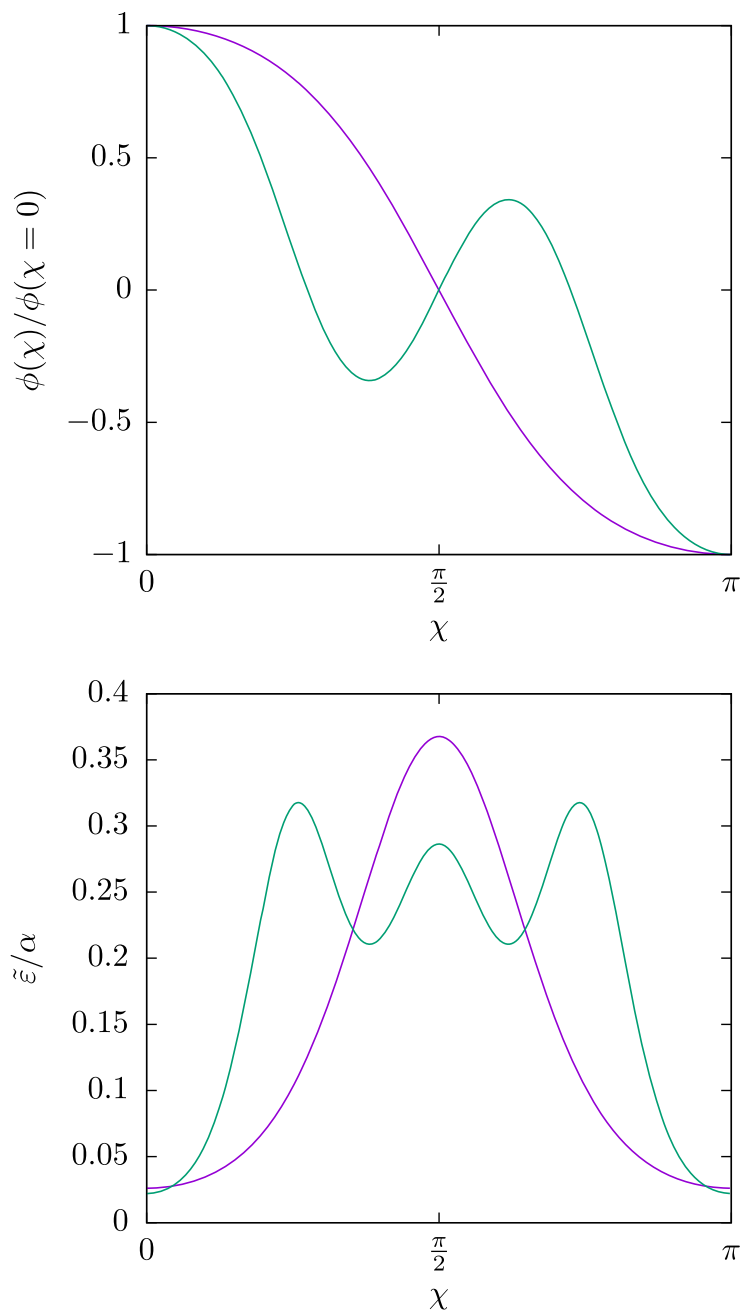

FIG. 5. We show the profile of a fundamental antikink solution (for $\alpha=5.0$, purple lines) together with a first excited antikink solution (for $\alpha=20.0$, green lines). We also give the energy density of these two solutions (right panel, same color coding).

solutions discussed above are linearly stable or unstable, and (b) the obtained spectra are important for comparing quantum fluctuations around the vacua of the model with those around solitons.

We use the following ansatz for the perturbations about the solutions $\phi(\chi)$ to Eq. (6):

$$
\Phi(\vec{r}, t)=\phi(\chi)+\Psi(\vec{r}, t)
$$

where $\Psi(\vec{r}, t)$ is a priori complex valued and we rescale $\Phi \rightarrow \eta \Phi$. The equation for $\Psi$ then reads

$$
\begin{aligned}
& \frac{1}{\sqrt{-g}} \partial_{\mu}\left(\sqrt{-g} \partial^{\mu} \Psi\right)-\alpha\left[\phi^{2}\left(2 \Psi+\Psi^{*}\right)-\Psi+\phi\left(2|\Psi|^{2}+\Psi^{2}\right)\right. \\
& \left.+|\Psi|^{2} \Psi\right]=0 .
\end{aligned}
$$

In the following, we will restrict our analysis to linear perturbations and hence neglect all terms of order $\mathcal{O}\left(\Psi^{2}\right)$ 
and higher. Note that these nonlinear terms would mix the modes of the linear spectrum, a fact well known from the Fermi-Pasta-Ulam-Tsingou problem [35]. We will use the following ansatz for the linear perturbations:

$\Psi(\vec{r}, t)=F_{1}(\chi, \theta, \varphi) \exp (-i \omega t)+F_{2}(\chi, \theta, \varphi) \exp (i \omega t)$,

where $F_{i}, i=1,2$ are assumed to be real-valued functions of the spatial coordinates and $\omega \in \mathbb{R}$ is chosen positive. Inserting this into the linearized version of Eq. (12), we find

$$
\begin{aligned}
& \frac{1}{\sqrt{-g}} \partial_{k}\left(\sqrt{-g} \partial^{k} F_{i}\right)-\alpha\left[\phi^{2}\left(2 F_{i}+F_{j}\right)-F_{i}\right] \\
& =-\omega^{2} F_{i}, \quad i, j=1,2, i \neq j, \quad k=1,2,3 .
\end{aligned}
$$

This clearly demonstrated that negative and positive frequency modes couple even for the vacuum, i.e., the absolute minimum of the theory with $\phi^{2} \equiv 1$. In the following, we will set $F_{1}=F_{2} \equiv F$ and use a product ansatz for $F$ that reads

$$
F(\chi, \theta, \varphi)=\psi(\chi) Y_{\ell \mu}(\theta, \varphi),
$$

where $Y_{\ell \mu}(\theta, \varphi), \ell=0,1,2,3, \ldots, \quad \mu=-\ell,-\ell+1, \ldots$, $\ell-1, \ell$ are the spherical harmonics. Using the rescalings $t \rightarrow t / R_{0}, \omega \rightarrow R_{0} \omega$, the equation for $\psi(\chi)$ then becomes

$\psi^{\prime \prime}+2 \cot \chi \psi^{\prime}-\alpha\left(3 \phi^{2}-1\right) \psi-\frac{\ell(\ell+1)}{\sin ^{2} \chi} \psi=-\omega^{2} \psi$,

where the prime here and in the following denotes the derivative with respect to $\chi$. To solve this equation and find the eigenfrequencies $\omega$, we introduce a new function via $\tilde{\psi}=\sin ^{-\ell}(\chi) \psi$ such that the equation reads

$\tilde{\psi}^{\prime \prime}+2(\ell+1) \cot \chi \tilde{\psi}^{\prime}-\alpha\left(3 \phi^{2}-1\right) \tilde{\psi}-\ell(\ell+2) \tilde{\psi}=-\omega^{2} \tilde{\psi}$.

We now have to employ appropriate boundary conditions. These are

$$
\tilde{\psi}^{\prime}(\chi=0)=\tilde{\psi}^{\prime}(\chi=\pi)=0,
$$

and without loss of generality we will construct solutions that have $\tilde{\psi}(\chi=0)=1$.

In the following, we will investigate the perturbations about the vacua as well as the kink and antikink solutions. Related to the question of stability of the solutions, we will investigate whether $\omega^{2}>0\left(\omega^{2}<0\right)$, which indicates that the solution is stable (unstable). We will also use the following notation: $\omega_{k, m, \ell}$ will denote the energy eigenvalues of the $k$ th excited perturbation with angular number $\ell$, i.e., a solution to Eq. (16) with $k$ nodes, of the scalar field solution of Eq. (4) with $m$ nodes associated with the $\ell$ th spherical harmonic. In this notation, $m=0$ corresponds to the vacua, $m=1$ the (anti)kink, $m=2$ to the kink and antikink, and higher $m$ to the excitations, respectively.

\section{A. Perturbations about the vacua $(m=0)$}

For the vacua $\psi(\chi) \equiv \pm 1$, i.e., the global minima of the potential, we obtain the equation

$$
\psi^{\prime \prime}+2 \cot \chi \psi^{\prime}-2 \alpha \psi-\frac{\ell(\ell+1)}{\sin ^{2} \chi} \psi=-\omega^{2} \psi .
$$

This equation is similar to that discussed in the case of a conformally coupled scalar field in an Einstein universe [24], and the general solutions to the equation are

$$
\psi_{k, \ell}(\chi)=\sin ^{\ell}(\chi) C_{k-\ell}^{\ell+1}(\cos \chi), \quad k=0,1,2, \ldots,
$$

where $C_{k-\ell}^{\ell+1}$ are the Gegenbauer functions and $\ell$ is now restricted by $\ell \leq k$. The corresponding discrete eigenfrequencies read

$$
\omega_{k, 0, \ell}^{2}=k(k+2)+2 \alpha, \quad k=0,1,2,3, \ldots,
$$

which do not depend on $\ell$. Hence, all multipoles have the same eigenfrequency, such that for a fixed value of $k$ the number of degenerate modes is $(k+1)^{2}$.

Note that $\omega_{k, 0, \ell}^{2}$ is always positive and always larger than $2 \alpha$. This indicates that the trivial solutions $\phi(\chi)= \pm 1$, i.e., the vacua, are stable.

\section{B. Perturbations about the (anti)kink $(m=1)$}

For the (anti)kink solutions discussed in Sec. III A, Eq. (16) has to be solved numerically. We find a discrete spectrum of modes that depends on $\alpha$. In the following, we will first discuss our results for the case $\ell=0$, i.e., the monopole contribution to the perturbations, and we will then comment on $\ell>0$, i.e., higher multipoles.

\section{The monopole $(\ell=0)$}

The lowest mode with $k=1$ has a profile for $\psi$ that resembles the (anti)kink solution itself, while higher modes, $k>1$, resemble the radially excited solutions discussed in Sec. III C. Note that a mode with $\psi \equiv$ constant $\neq 0$ does not exist in this case, as is apparent from Eq. (16); i.e., the first mode fulfilling the periodic boundary conditions is a solution with one node, $k=1$.

For $\alpha \rightarrow \alpha_{\min , 1}=3$, we find $\omega_{1,1,0}^{2} \rightarrow 0, \omega_{2,1,0}^{2} \rightarrow 5$, $\omega_{3,1,0}^{2} \rightarrow 12$, which indicates that

$$
\omega_{k, 1,0}^{2} \rightarrow k(k+2)-3 \text { for } \alpha \rightarrow 3 .
$$

Moreover, Fig. 6 (left panel) demonstrates that the value of $\omega_{k, 1,0}^{2}$ increases when we increase $\alpha$ from its limiting value 

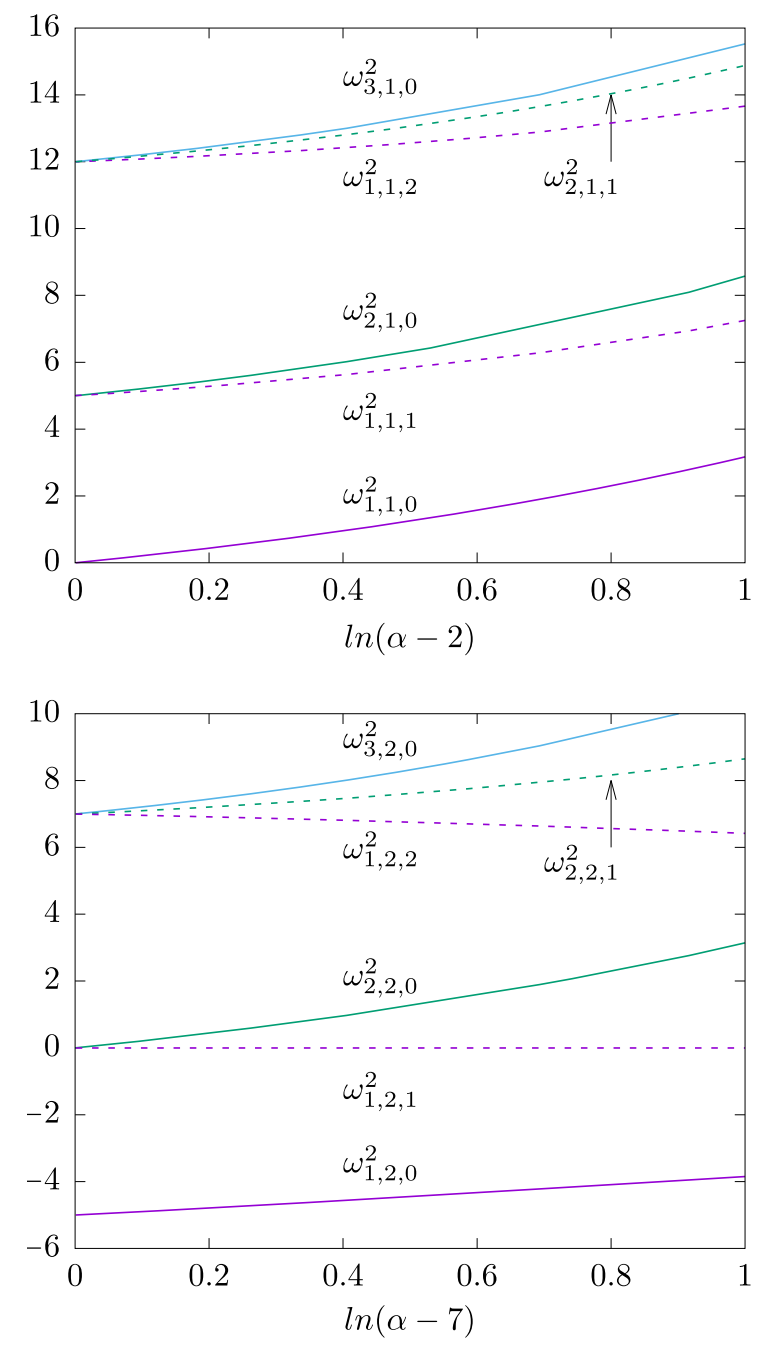

FIG. 6. We show the lowest eigenfrequencies $\omega_{k, m, \ell}^{2}$ of the $k$ th perturbation about the $m$ th soliton with angular quantum number $\ell$ in dependence on $\alpha$. (Left panel) The value of $\omega_{k, 1,0}^{2}$ for the monopole $(\ell=0)$ perturbations (solid lines) about the (anti)kink solution $(m=1)$ for $k=1,2,3$. We also give the value of $\omega_{k, 1,1}^{2}$ of the dipole $(\ell=1)$ perturbation for $k=1,2$ and $\omega_{1,1,2}^{2}$ of the quadrupole ( $\ell=2$ ) perturbation for $k=1$ (dashed lines). (Right panel) The values of $\omega_{k, 2,0}^{2}$ for the monopole $(\ell=0)$ perturbation about the kink-antikink solution $(m=2)$ for $k=1,2,3$ (solid lines). We also give the value of $\omega_{k, 2,1}^{2}$ of the dipole $(\ell=1)$ perturbation for $k=1,2$ and $\omega_{1,2,2}^{2}$ of the quadrupole $(\ell=2)$ perturbation for $k=1$ (dashed lines).

$\alpha_{\min , 1}=3$. Hence, the eigenvalues $\omega_{k, 1,0}^{2}$ of all $\ell=0$ perturbations about the (anti)kink solution are positive.

\section{The dipole $(\ell=1)$ and quadrupole $(\ell=2)$}

We have studied the dipole $(\ell=1)$ and quadrupole $(\ell=2)$ contribution for different values of $k$. Our results for the lowest modes are shown in Fig. 6. As is obvious from this figure, the degeneracy of the eigenfrequencies with respect to the angular number $\ell$ no longer exists in the presence of the (anti)kink. We find that $\omega_{k, 1, \ell}^{2}$ for a fixed $k$ increases with increasing $\ell$; see the values for $\omega_{1,1, \ell}^{2}$ (dashed lines). Moreover, the eigenfrequencies $\omega_{1,1,2}^{2}$ are comparable in value to the eigenfrequencies $\omega_{2,1,1}^{2}$ and $\omega_{3,1,0}^{2}$, and in the limit $\alpha \rightarrow \alpha_{\min , 1}=3$ they tend to the same value. This suggests that for a fixed $m$, the eigenfrequencies $\omega_{k, m, \ell}^{2}$ with $k+\ell$ constant are comparable in value and degenerate for $\alpha \rightarrow \alpha_{\min , m}$. Similar to the monopole discussed above, the eigenfrequencies for all $k$ and $\ell$ that we have studied are positive, and since their values increase with both $k$ and $\ell$, we find that the (anti)kink is linearly stable under general perturbations.

\section{Perturbations about the kink and antikink $(m=2)$}

Again, we will first discuss the monopole perturbation, $\ell=0$, then comment on $\ell>0$.

\section{The monopole $(\ell=0)$}

In Fig. 6 (right panel), we give the dependence of the eigenvalues $\omega_{k, 2,0}^{2}, k=1,2,3$ on $\alpha$. In contrast to the perturbations about the vacuum and the (anti)kink, respectively, we find one mode with a negative eigenvalue. This is the lowest mode, $k=1$, for which the profile $\psi_{1}$ possesses one node and eigenvalue $\omega_{1,2,0}^{2}<0$. For $\alpha \rightarrow \alpha_{\min , 2}=8$, we find that $\omega_{1,2,0}^{2} \rightarrow-5$. This eigenvalue increases when $\alpha$ increases, but it stays negative for all values of $\alpha$ that we have investigated. We find, e.g., that $\omega_{1,2,0}^{2} \approx-2.12$ for $\alpha=100$, and our numerical results further indicate that $\omega_{1,2,0}^{2} \rightarrow 0$ for $\alpha \rightarrow \infty$. On the other hand, the eigenvalues of higher modes - in Fig. 6 (right panel) shown for $k=2,3$ are positive for all values of $\alpha \geq \alpha_{\min , 2}=8$ with $\omega_{2,2,0}^{2} \rightarrow 0$ for $\alpha \rightarrow \alpha_{\min , 2}$. We conclude that the kink-antikink solution has (at least) one unstable mode and hence is linearly unstable.

\section{The dipole $(\ell=1)$ and quadrupole $(\ell=2)$}

Considering the dipole and quadrupole contributions of the perturbations, we find again that the degeneracy with respect to $\ell$ no longer exists in the presence of the kink and antikink. Moreover, a few other interesting things appear. The first thing to note is that the dipole contribution of the $k=1$ perturbation about the kink and antikink has eigenfrequency $\omega_{1,2,1}^{2} \equiv 0$ for all values of $\alpha$ that we have studied. Hence, this perturbations corresponds to a zero mode of the system. Moreover, similar to the (anti)kink case, the eigenfrequencies $\omega_{3,2,0}^{2}, \omega_{2,2,1}^{2}$ and $\omega_{1,2,2}^{2}$ are comparable in value and become degenerate in value for $\alpha \rightarrow \alpha_{\min , 2}=8$. This strengthens the claim made above for $m=1$ that for a fixed $m$, the eigenfrequencies $\omega_{k, m, \ell}^{2}$ with $k+\ell$ constant are comparable in value and degenerate for $\alpha \rightarrow \alpha_{\min , m}$. There is one further interesting point that is new in comparison to perturbations about the (anti)kink. 
We find that the eigenfrequency $\omega_{1,2,2}^{2}$, i.e., the quadrupole contribution to the $k=1$ perturbations has decreasing values for $\alpha$ increasing - in contrast to all other perturbations that we have discussed so far.

\section{Perturbations for $m>2$ and/or $\ell>2$ and/or $k>3$}

To strengthen the claims about the eigenfrequency spectra, we have studied several other values of $k, m, \ell$. The values of $\omega_{k, m, \ell}^{2}$ for $\alpha=\alpha_{\min , m}+1$ and $\alpha=\alpha_{\min , m}+2$, respectively, and all possible combinations for $k \in[1: 3]$, $m \in[1: 3]$ and $\ell \in[0: 3]$ are given in Table I. For the perturbations about the (anti)kink $(m=1)$, we find that

TABLE I. The value of $\omega_{k, m, \ell}^{2}$ for different choices of $m, k, \ell$ at $\alpha_{\min , m}+1$ and $\alpha_{\min , m}+2$, respectively. For $m=1, m=2$, $m=3$, the value is $\alpha_{\min , 1}=3.0, \alpha_{\min , 2}=8.0, \alpha_{\min , 3}=15.0$, respectively.

\begin{tabular}{|c|c|c|c|c|}
\hline$m$ & $k$ & $\ell$ & $\begin{array}{l}\omega_{k, m, \ell}^{2} \text { for } \\
\alpha_{\min , m}+1\end{array}$ & $\begin{array}{c}\omega_{k, m, \ell}^{2} \text { for } \\
\alpha_{\min , m}+2 \\
\end{array}$ \\
\hline 1 & 1 & 0 & 1.90 & 3.65 \\
\hline 1 & 1 & 1 & 6.28 & 7.61 \\
\hline 1 & 2 & 0 & 7.05 & 9.14 \\
\hline 1 & 1 & 2 & 12.89 & 13.91 \\
\hline 1 & 2 & 1 & 13.65 & 15.38 \\
\hline 1 & 3 & 0 & 14.01 & 16.02 \\
\hline 1 & 1 & 3 & 21.61 & 22.40 \\
\hline 1 & 2 & 2 & 22.33 & 23.79 \\
\hline 1 & 3 & 1 & 22.77 & 24.58 \\
\hline 1 & 2 & 3 & 33.08 & 34.32 \\
\hline 1 & 3 & 2 & 33.54 & 35.16 \\
\hline 1 & 3 & 3 & 46.33 & 47.79 \\
\hline 2 & 1 & 0 & -4.22 & -3.74 \\
\hline 2 & 1 & 1 & 0 & 0 \\
\hline 2 & 2 & 0 & 1.89 & 3.59 \\
\hline 2 & 1 & 2 & 6.64 & 6.34 \\
\hline 2 & 2 & 1 & 7.95 & 8.93 \\
\hline 2 & 3 & 0 & 9.04 & 11.10 \\
\hline 2 & 1 & 3 & 15.46 & 14.99 \\
\hline 2 & 2 & 2 & 16.36 & 16.86 \\
\hline 2 & 3 & 1 & 17.36 & 18.78 \\
\hline 2 & 2 & 3 & 26.99 & 27.14 \\
\hline 2 & 3 & 2 & 27.83 & 28.79 \\
\hline 2 & 3 & 3 & 40.43 & 41.03 \\
\hline 3 & 1 & 0 & -11.66 & -11.52 \\
\hline 3 & 1 & 1 & -7.24 & -7.47 \\
\hline 3 & 2 & 0 & -5.95 & -5.23 \\
\hline 3 & 1 & 2 & -0.39 & -0.75 \\
\hline 3 & 2 & 1 & 0 & 0 \\
\hline 3 & 3 & 0 & 1.91 & 3.62 \\
\hline 3 & 2 & 2 & 8.56 & 8.18 \\
\hline 3 & 1 & 3 & 8.60 & 8.20 \\
\hline 3 & 3 & 1 & 9.76 & 10.54 \\
\hline 3 & 2 & 3 & 19.41 & 18.85 \\
\hline 3 & 3 & 2 & 20.07 & 20.25 \\
\hline 3 & 3 & 3 & 32.68 & 32.48 \\
\hline
\end{tabular}

higher $\ell$ follow the pattern identified before. For all values of $k, \ell$, we find that $\omega_{k, 1, \ell}^{2}$ is positive and increases with $\alpha$.

When considering perturbations about the kink and antikink $(m=2)$, we find that the eigenfrequencies for $k+\ell=$ constant have the lowest value for the highest possible $\ell$. This indicates that multipole perturbations along the $\theta, \varphi$ directions on the $S^{3}$ cost less energy than the perturbations in the $\chi$-direction. We also find that for $\ell>k$ the eigenfrequency decreases with increasing $\alpha$.

A very similar pattern to that described above appears for the case $m=3$; however, there are some small differences. First, we find a number of negative eigenfrequencies:
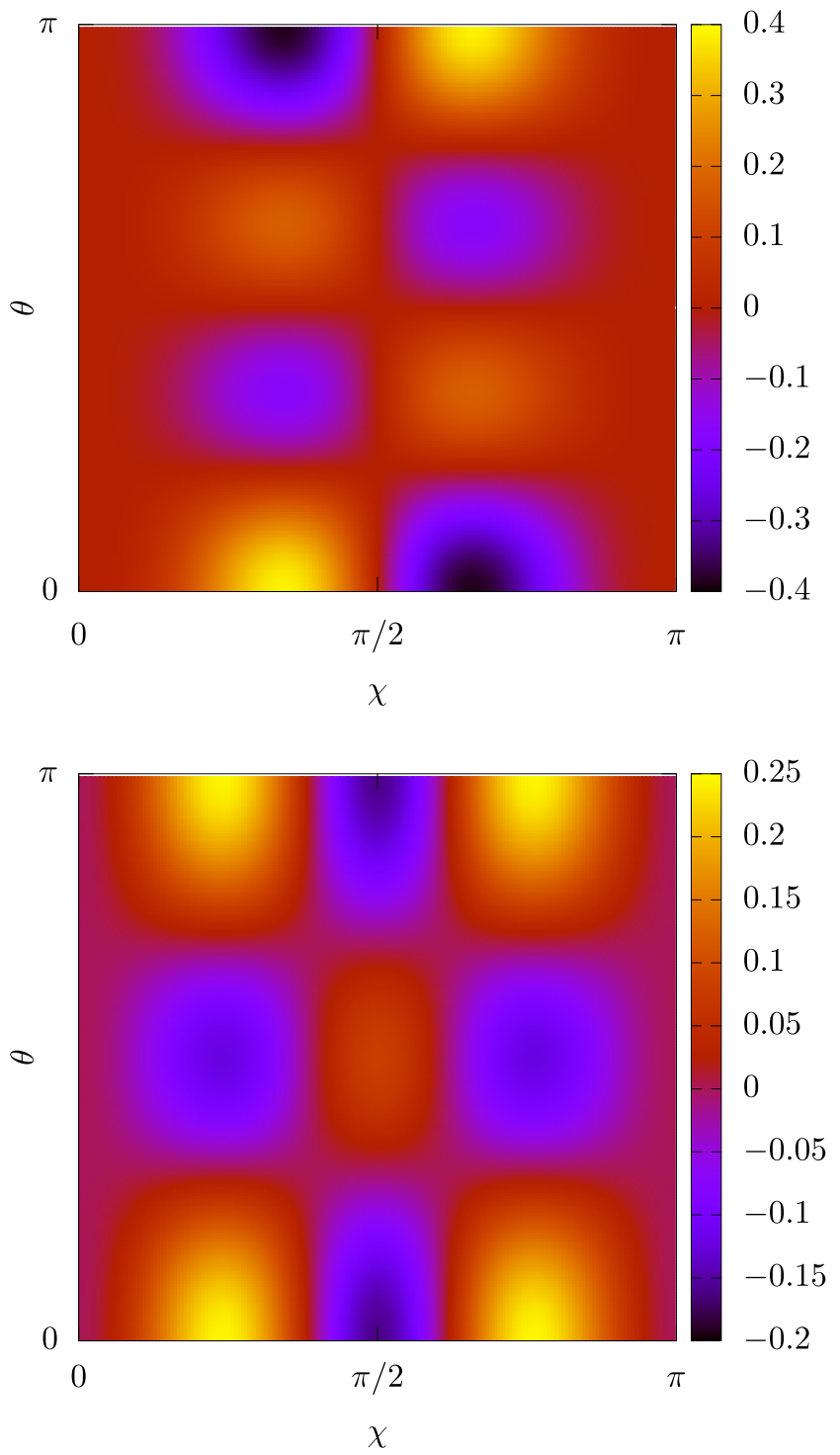

FIG. 7. We show the value of $|\psi|$ of the perturbations about the kink solution $(m=1)$ for $\alpha=4$. (Left panel) The octupole contribution $(\ell=3)$ to the first perturbation $(k=1)$ which has $\omega_{1,1,3}^{2} \approx 21.61$. (Right panel) The quadrupole contribution $(\ell=2)$ to the second perturbation $(k=2)$ which has $\omega_{2,1,2}^{2} \approx 22.33$. 
$\omega_{k, 3, \ell}^{2} \leq 0$ for $k<m$ and $\ell<m$ and the equality holding for $k=m-1=2, \ell=1$. Moreover, the statement that for $k+\ell$ constant the highest $\ell$ has the lowest eigenfrequency is true only for $k+\ell \leq 3$. For $k+\ell=4$, we find that the $k=\ell=2$ perturbation has a lower eigenfrequency value than the $k=1, \ell=3$ perturbation. Moreover, all perturbations with $\ell \geq k$ now have a decreasing value of the eigenfrequency when $\alpha$ increases.

In Fig. 7, we show $|\psi|$ for two perturbations about the (anti)kink solution for $k+\ell=$ constant-in this case, $k+\ell=4$. We have chosen the octupole contribution $(\ell=3)$ of the first excitation $(k=1)$ as well as the quadrupole contribution $(\ell=2)$ of the second excitation $(k=2)$. These perturbations are obviously very different in nature, but they have comparable eigenfrequencies at $\alpha=4$.

\section{CONCLUSIONS}

We have demonstrated that the no-go theorem for real, static, stable scalar field solitons in $(3+1)$ dimensions in a curved space-time background do not extend to a spacetime that is spatially compact. We have constructed (anti)kink and kink-antikink solutions in an Einstein universe background and have shown that the (anti)kink solutions are linearly stable. Moreover, $m$-excited solutions do exist that possess $m$ nodes in the scalar field profile.

The study of the linear perturbations about the solutions has shown a number of interesting features. The degeneracy with respect to the angular number $\ell$ that is present for the perturbations about the vacua no longer exists in the presence of the solitoniclike objects. Moreover, we find that for an $m$-kink the eigenfrequencies are associated with the perturbations $\omega_{k, m, \ell}^{2} \leq 0$, hence indicating instability, whenever $k<m$ and-at the same time- $\ell<m$. Since the lowest order perturbation about the (anti)kink $(m=1)$ is $k=1$, there are no negative eigenfrequency perturbations and the (anti)kink is linearly stable. For the particular case in which $k=m-1$ and $\ell=1$, we find that the corresponding eigenfrequency $\omega_{m-1, m, 1}^{2}$ has value zero. Hence, the corresponding perturbations correspond to zero modes of the model.

The eigenfrequency spectra and the corresponding perturbations $\psi$ are important in the computation of quantum effects in given space-times, especially in those with a nonvanishing curvature; see, e.g., [24,25]. Compact spacetimes are often considered, rendering the eigenfrequency spectra discrete. Here, we have studied the example of a positively curved, spatially compact space-time given by the Einstein universe. While this static universe is not the Universe we observe, it has features similar to the latter: it contains a perfect fluid with positive energy density and vanishing pressure (ordinary matter) as well as a positive cosmological constant. In computations of quantum effects, it is often considered a good approximation to the observed Universe in sufficiently small time intervals. What we have demonstrated in this paper is that the presence of solitoniclike objects [in the form of (anti)kinks] modifies the eigenfrequency spectra of a possible quantum perturbation (given by the field $\psi$ ) considerably. Since the Universe is supposed to have gone through a number of phase transitions in the early Universe during which topological defects, i.e., localized solitoniclike objects, (could) have formed, computations of quantum effects (e.g., the Casimir effect) should certainly take these into account.

Let us finally remark that in this paper we have only studied static solutions and have demonstrated (see the Appendix) that a full backreacted solution with space-time symmetries equivalent to that of the Einstein universe cannot be static. However, one can consider the nonlinear dynamics of the scalar field itself on a static space-time background. The first possibility would then be to study the dynamical decay of the unstable solutions in our model and get an estimate for the scalar radiation emitted in this process. Moreover, a simulation of the scattering of the solutions in our model would be interesting to see how the fact that the background is not (asymptotically) Minkowski will influence the scattering properties of the diverse solutions. Since the model at hand is not integrable, we would expect internal modes to be excited in the scattering. Moreover, the study of the nonlinear dynamics of the full backreacted system would be interesting to understand how the stability of the solutions is influenced by a dynamical space-time and how and whether - next to scalar radiation-gravitational radiation would be emitted in the decay or scattering of the solitons. This is currently under investigation.

\section{ACKNOWLEDGMENTS}

B. H. thanks FAPESP for the financial support from Grant No. 2019/01511-5. G. L. thanks FAPES for the financial support from EDITAL CNPq/FAPES Grant No. 22/2018. C. F. S. P. thanks FAPES for the financial support from Grant No. 98/2017. We thank FAPES for the support from Grant No. 0447/2015.

\section{APPENDIX: NO STATIC UNIVERSE FOR SCALAR MATTER}

Let us assume the metric to be of the FLRW type,

$d s^{2}=-d t^{2}+R(t)^{2}\left[d \chi^{2}+\sin ^{2} \chi\left(d \theta^{2}+\sin ^{2} \theta d \varphi^{2}\right)\right]$,

such that the nonvanishing components of the Einstein tensor read

$$
\begin{aligned}
G_{t t} & =3\left(\frac{\dot{R}^{2}}{R^{2}}+\frac{1}{R^{2}}\right), \quad G_{\chi \chi}=-\left(2 \ddot{R} R+\dot{R}^{2}+1\right), \\
G_{\theta \theta} & =\sin ^{2} \chi G_{\chi \chi}, \quad G_{\varphi \varphi}=\sin ^{2} \chi \sin ^{2} \theta G_{\chi \chi},
\end{aligned}
$$


and the dot denotes the derivative with respect to $t$. Using the components of the energy-momentum tensor of a static scalar field given in Eq. (4) and replacing $R_{0} \rightarrow R(t)$, the relevant components of the Einstein equation $G_{\mu \nu}+$ $\Lambda g_{\mu \nu}=8 \pi G T_{\mu \nu}$ are

$$
3\left(\frac{\dot{R}^{2}}{R^{2}}+\frac{1}{R^{2}}\right)-\Lambda=8 \pi G\left[\frac{1}{2} \frac{\phi^{\prime 2}}{R^{2}}+V(\phi)\right],
$$

$$
-\left(2 \frac{\ddot{R}}{R}+\frac{\dot{R}^{2}}{R^{2}}+\frac{1}{R^{2}}\right)+\Lambda=8 \pi G\left[\frac{1}{2} \frac{\phi^{\prime 2}}{R^{2}}-V(\phi)\right] .
$$

Combining these two equations for the case of a static universe, $\dot{R}=\ddot{R} \equiv 0$, we find that $4 \pi G \phi^{\prime 2}=1$, which implies $\phi(\chi) \sim \chi+$ constant. This solution is not compatible with the periodicity condition, and hence no static universe exists when choosing a static scalar field as the given matter source.
[1] S. Chatrchyan et al. (CMS Collaboration), Observation of a new boson at a mass of $125 \mathrm{GeV}$ with the CMS experiment at the LHC, Phys. Lett. B 716, 30 (2012).

[2] G. Aad et al. (ATLAS Collaboration), Observation of a new particle in the search for the Standard Model Higgs boson with the ATLAS detector at the LHC, Phys. Lett. B 716, 1 (2012).

[3] F. Englert and R. Brout, Broken Symmetry and the Mass of Gauge Vector Mesons, Phys. Rev. Lett. 13, 321 (1964).

[4] P. Higgs, Broken Symmetries and the Masses of Gauge Bosons, Phys. Rev. Lett. 13, 508 (1964).

[5] G. Guralnik, C. R. Hagen, and T. W. B. Kibble, Global Conservation Laws and Massless Particles, Phys. Rev. Lett. 13, 585 (1964).

[6] V. L. Ginzburg and L. D. Landau, On the theory of superconductivity, Zh. Eksp. Teor. Fiz. 20, 1064 (1950).

[7] N. S. Manton, Solitons as elementary particles: A paradigm scrutinized, Nonlinearity 21, T221 (2008).

[8] F. Lund and T. Regge, Tullio, Unified approach to strings and vortices with soliton solutions, Phys. Rev. D 14, 1524 (1976).

[9] N. S. Manton and P. M. Sutcliffe, Topological Solitons, Cambridge Monographs on Mathematical Physics (Cambridge University Press, Cambridge, England, 2004).

[10] G. H. Derrick, Comments on nonlinear wave equations as models for elementary particles, J. Math. Phys. (N.Y.) 5, 1252 (1964).

[11] P. Bowcock, D. Foster, and P. M. Sutcliffe, $Q$-balls, integrability and duality, J. Phys. A 42, 085403 (2009).

[12] T. D. Lee and Y. Pang, Nontopological solitons, Phys. Rep. 221, 251 (1992).

[13] S. R. Coleman, $Q$ balls, Nucl. Phys. B262, 263 (1985); Erratum, Nucl. Phys. B269, 744(E) (1986).

[14] M. S. Volkov and E. Wöhnert, Spinning $Q$ balls, Phys. Rev. D 66, 085003 (2002).

[15] B. Kleihaus, J. Kunz, M. List, and I. Schaffer, Rotating boson stars and $Q$-balls. II. Negative parity and ergoregions, Phys. Rev. D 77, 064025 (2008).

[16] B. Kleihaus, J. Kunz, and M. List, Rotating boson stars and $Q$-balls, Phys. Rev. D 72, 064002 (2005).
[17] D. J. Kaup, Klein-Gordon geon, Phys. Rev. 172, 1331 (1968).

[18] R. Ruffini and S. Bonazzola, Systems of selfgravitating particles in general relativity and the concept of an equation of state, Phys. Rev. 187, 1767 (1969).

[19] F. E. Schunck and E. W. Mielke, Boson stars: Rotation, formation, and evolution, Gen. Relativ. Gravit. 31, 787 (1999).

[20] R. Friedberg, T. D. Lee, and Y. Pang, Mini-soliton stars, Phys. Rev. D 35, 3640 (1987).

[21] P. Jetzer, Boson stars, Phys. Rep. 220, 163 (1992).

[22] S. Carloni and J. L. Rosa, Derrick's theorem in curved spacetime, Phys. Rev. D 100, 025014 (2019).

[23] J. A. Gonzalez and D. Sudarsky, Scalar solitons in a 4-dimensional curved space-time, Rev. Mex. Fis. 47, 231 (2001).

[24] L. H. Ford, Quantum vacuum energy in general relativity, Phys. Rev. D 11, 3370 (1975).

[25] L. H. Ford, Quantum vacuum energy in a closed universe, Phys. Rev. D 14, 3304 (1976).

[26] L. H. Ford, Casimir effect for a self-interacting scalar field, Proc. R. Soc. A 368, 305 (1979).

[27] C. A. R. Herdeiro and M. Sampaio, Casimir energy and a cosmological bounce, Classical Quantum Gravity 23, 473 (2006).

[28] M. B. Altaie, Back reaction of quantum fields in an Einstein universe, Phys. Rev. D 65, 044028 (2002).

[29] M. B. Altaie and E. Malkawi, Bose-Einstein condensation of spin-1 field in an Einstein universe, J. Phys. A 33, 7093 (2000).

[30] L. Parker and Y. Zhang, Ultrarelativistic Bose-Einstein condensation in the Einstein universe and energy conditions, Phys. Rev. D 44, 2421 (1991).

[31] M. B. Altaie and J. S. Dowker, Spinor fields in an Einstein universe: Finite-temperature effects, Phys. Rev. D 18, 3557 (1978).

[32] A. Einstein, Kosmologische Betrachtungen zur allgemeinen Relativitätstheorie, Sitzungsber. K. Preuss. Akad. Wiss. 1917, 142152 (1917). 
[33] Y. Brihaye and C. Gabriel, Skyrme model on S(3) and harmonic maps, Mod. Phys. Lett. A 14, 893 (1999).

[34] U. Ascher, J. Christiansen, and R. D. Russell, A collocation solver for mixed order systems of boundary value problems, Math. Comput. 33, 659 (1979); Collocation software for boundary-value ODEs, ACM Trans. Math. Softw. 7, 209 (1981).

[35] E. Fermi, J. Pasta, and S. Ulam, Studies of nonlinear problems, Los Alamos National Laboratory, 1955, http:// www.physics.utah.edu/detar/phys6720/handouts/fpu/ FermiCollectedPapers 1965.pdf. 